University of Wollongong

Research Online

Faculty of Engineering and Information

Faculty of Engineering and Information

Sciences - Papers: Part A

Sciences

$1-1-2012$

\title{
Fairness and capacity analysis of opportunistic feedback protocol with proportional fair or maximum throughput scheduling
}

\author{
Hang Li \\ University Of Western Australia \\ Qinghua Guo \\ University of Wollongong, qguo@uow.edu.au \\ Licai Fang \\ University Of Western Australia \\ Defeng (David) Huang \\ University Of Western Australia, david.huang@uwa.edu.au
}

Follow this and additional works at: https://ro.uow.edu.au/eispapers

Part of the Engineering Commons, and the Science and Technology Studies Commons

Research Online is the open access institutional repository for the University of Wollongong. For further information contact the UOW Library: research-pubs@uow.edu.au 


\title{
Fairness and capacity analysis of opportunistic feedback protocol with proportional fair or maximum throughput scheduling
}

\author{
Abstract \\ An opportunistic feedback protocol with maximum throughput (MT) scheduling has been proposed in the \\ literature to achieve multiuser diversity for the downlink transmission of a wireless system, and its system \\ capacity has been analyzed with the assumption that the received signal-to-noise ratios (SNRs) for the \\ users are independent and identically distributed. In this paper, fairness and capacity performances of the \\ opportunistic feedback protocol are analyzed for SNRs with independent but not necessarily identical \\ distributions. In addition to MT scheduling, we also analyze the fairness and capacity performances for \\ proportional fair (PF) scheduling. Compared with MT scheduling, numerical results demonstrate that the \\ PF scheduling achieves strict fairness at the cost of about $10 \%$ system capacity loss over Rayleigh fading \\ channels. Moreover, the feedback threshold and random access probability of the opportunistic feedback \\ protocol are jointly optimized using a constrained gradient descent method to maximize system capacity.

\section{Keywords} \\ opportunistic, feedback, protocol, proportional, fair, maximum, capacity, throughput, analysis, scheduling, \\ fairness \\ Disciplines \\ Engineering | Science and Technology Studies

\section{Publication Details} \\ H. Li, Q. Guo, L. Fang \& D. Huang, "Fairness and capacity analysis of opportunistic feedback protocol with \\ proportional fair or maximum throughput scheduling," in International Conference on Wireless \\ Communications and Signal Processing, 2012, pp. 1-5.
}




\title{
Fairness and Capacity Analysis of Opportunistic Feedback Protocol with Proportional Fair or Maximum Throughput Scheduling
}

\author{
Hang Li*, Qinghua Guo*†, Licai Fang* and Defeng (David) Huang* \\ ${ }^{*}$ School of Electrical, Electronic and Computer Engineering, \\ The University of Western Australia, 35 Stirling Highway, Crawley, WA 6009, Australia \\ ${ }^{\dagger}$ School of Electrical, Computer and Telecommunications Engineering, \\ University of Wollongong, NSW 2522, Australia \\ Email: hangli@ee.uwa.edu.au, qinghua_guo@uow.edu.au, licaifang@gmail.com, david.huang@uwa.edu.au
}

\begin{abstract}
An opportunistic feedback protocol with maximum throughput (MT) scheduling has been proposed in the literature to achieve multiuser diversity for the downlink transmission of a wireless system, and its system capacity has been analyzed with the assumption that the received signal-to-noise ratios (SNRs) for the users are independent and identically distributed. In this paper, fairness and capacity performances of the opportunistic feedback protocol are analyzed for SNRs with independent but not necessarily identical distributions. In addition to MT scheduling, we also analyze the fairness and capacity performances for proportional fair (PF) scheduling. Compared with MT scheduling, numerical results demonstrate that the PF scheduling achieves strict fairness at the cost of about $10 \%$ system capacity loss over Rayleigh fading channels. Moreover, the feedback threshold and random access probability of the opportunistic feedback protocol are jointly optimized using a constrained gradient descent method to maximize system capacity.
\end{abstract}

Index Terms-Multiuser diversity, opportunistic feedback, system capacity, average system fairness.

\section{INTRODUCTION}

Significant system capacity increase can be achieved by exploiting multiuser diversity in a wireless network, where the channel is always scheduled to the user with the best channel quality at any given time [1], [2]. Maximum throughput (MT) scheduling maximizes system capacity without any fairness consideration, whereas proportional fair (PF) scheduling achieves fairness among all users at the expense of system capacity loss [3]. However, both schedulings require the channel quality information (CQI, e.g., the received signal to noise ratio (SNR) or alternative channel quality indicator) of each user to be fed back to the Base Station (BS), inducing considerable feedback overhead which may overshadow the multiuser diversity gain. For example, in a full-feedback protocol, the amount of feedback overhead through dedicated feedback channels increases linearly with the number of users, $N$, while the total throughput, under Rayleigh fading channels, grows $O(\log \log N)$ [4].

A variety of approaches such as those in [5]-[7], have been proposed to reduce the feedback overhead, but the feedback

This work was supported by Australian Research Council's Discovery Projects DP0877616 and DP1093000, and DECRA Grant DE120101266. overhead still increases linearly with $N$. To further reduce feedback overhead, an opportunistic feedback protocol [8] in terms of MT scheduling was proposed for contentionbased feedback channel with a fixed number of feedback minislots, where system capacity is maximized by optimizing the parameters of channel power threshold and random access probability. In [9], the capture effect on system capacity was analyzed for the protocol. However, in [8] and [9], only system capacity performance was evaluated, and the analysis was carried out only for MT scheduling, where all users' channels were assumed to follow independent and identically distributed fading.

On the other hand, another protocol in [10] analyzed the capacity and fairness performances in terms of PF and MT schedulings, where the BS terminates the remaining feedback process by broadcasting a termination message when receiving a feedback message successfully. However, for the protocol in [10], before all active users stop the feedback process, some time delay will be incurred due to the signal propagation delay and the necessary signal processing time. As a result, the protocol of [10] can be less efficient than the protocol of [8] when the BS covers a relatively large geographical area. In addition, due to the variable feedback times, the synchronization among all active users and the BS may become a serious problem (e.g., the termination message may get lost). In contrast, the synchronization for the protocol of [8] is much easier since the time for the feedback process is fixed and there is no termination packet from the BS. As a result, in this paper, we will focus on the protocol proposed in [8].

In this paper, the performances of both average system fairness and system capacity are analyzed for the opportunistic feedback protocol with PF or MT scheduling, in terms of the probability density distributions of the users' SNRs (the SNRs are assumed to be independent but not necessarily identically distributed). The analytical method is different from that of [10]. In our work, we focus on the probabilities that are pertained to a particular user (i.e. the $n$th user), instead of a group of users in [10]. Numerical results show that the PF scheduling for the opportunistic feedback protocol achieves 
fairness at the cost of a limited system capacity loss with respect to the MT scheduling over Rayleigh fading channels.

In the opportunistic feedback protocol, the selection of the parameter values (i.e., the feedback threshold and random access probability) has a significant impact on system capacity. Using extensive simulations, we will show that the constrained gradient descent method [11] can be used to find the optimal values of the two parameters.

The remainder of this paper is organized as follows: Section II describes the system model of the PF or MT opportunistic feedback protocol. Section III shows the fairness and capacity performance analysis of the protocol. Section IV presents the numerical results and finally, Section $\mathrm{V}$ concludes the paper.

\section{SYSTEM MODEL}

\section{A. Downlink Transmission Model}

We consider the downlink of a Frequency Division Duplexing system with one BS and $N$ active users. As in [8], we make the following assumptions: (1) All active users are ideally synchronized to the BS. (2) The data for active users is infinitely backlogged; therefore the queueing dynamics is not considered. (3) The channel is frequency flat fading, blockwise time-invariant for each frame and changes independently from frame to frame. However, in this paper the fading channel gains of different users are assumed to be independent but not necessarily identically distributed.

\section{B. Uplink Feedback}

Fig. 1 shows the framing structure of the opportunistic feedback protocol. The BS first informs all active users to respond by a broadcast message ${ }^{1}$. Using the broadcast message, all users synchronize with the BS and estimate their SNRs. Then, only the users whose scheduling metrics are greater than their feedback thresholds, feed back a packet carrying their user identity (UID) information with a random access probability $p$ in $K$ uplink random access minislots. It is assumed that if more than two packets collide in the same minislot, none of the feedback packets can be decoded. The random access attempts are assumed to be independent among users and minislots. If there is more than one successful feedback user in the $K$ minislots, the BS randomly selects one of them. If there is no successful feedback user in any minislot, the BS randomly selects one user among all the $N$ active users. Once a user is selected, the BS polls and requests it to report its CQI. Finally, using the CQI feedback, the BS selects an appropriate transmission rate which is assumed to be the downlink channel capacity.

\section{Scheduling Metric}

In the PF and MT schedulings, the scheduler selects the user with the largest value of [3], [12]

$$
\Gamma_{i}= \begin{cases}\gamma_{i} / \bar{\gamma}_{i}, & \text { PF scheduling } \\ \gamma_{i}, & \text { MT scheduling }\end{cases}
$$

\footnotetext{
${ }^{1}$ The broadcast message includes system configuration information (e.g. the values of the feedback threshold, the random access probability and the number of minislots).
}

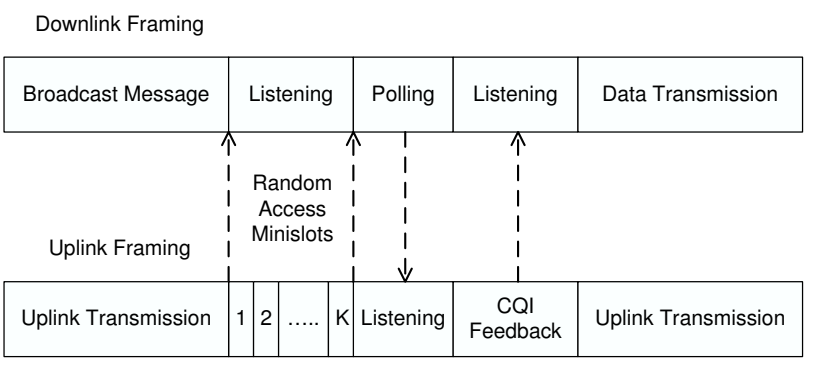

Fig. 1. The framing structure of the opportunistic feedback protocol.

where $\gamma_{i}$ and $\bar{\gamma}_{i}$ are the instantaneous and average received SNRs of user $i$, respectively.

\section{Fairness Measure}

It is assumed that all users are equally important and have the same quality of service requirements. The self-fairness of user $i$ is defined as [3], [13], [14]

$$
\zeta_{i}=\frac{-\log \left(P_{i}\right)}{\log (N)}
$$

where $P_{i}$ is the proportion of resources allocated to user $i$, or equivalently the probability of user $i$ being selected, and $\log (N)$ is a normalization factor. The average system fairness is then defined as [3], [13], [14]

$$
\zeta=\sum_{i=1}^{N} P_{i} \zeta_{i}=-\sum_{i=1}^{N} P_{i} \frac{\log \left(P_{i}\right)}{\log (N)} .
$$

Note that a system is strictly fair if $P_{i}=1 / N, \forall i \in$ $\{1,2, \ldots, N\}$, regardless of their average SNRs, and the average system fairness is 1 .

\section{FAIRNESS AND CAPACITY ANALYSIS}

\section{A. PF Scheduling}

All users are assumed to use the same feedback threshold $\eta$ and the same random access probability $p$. Let $P_{n, 0}$ denote the probability that user $n$ 's scheduling metric $\Gamma_{n}<\eta$ and user $n$ is selected, and $P_{n, 1}$ denote the probability that $\Gamma_{n} \geq \eta$ and user $n$ is selected. The average system fairness is then given by

$$
\zeta=-\sum_{n=1}^{N}\left(P_{n, 0}+P_{n, 1}\right) \frac{\log \left(P_{n, 0}+P_{n, 1}\right)}{\log (N)}
$$

Let $f_{\gamma_{n}}(x)$ and $F_{\gamma_{n}}(x)$ be the probability density function (pdf) and the cumulative distribution function (cdf) of $\gamma_{n}$, respectively. The system capacity can then be given by

$$
\begin{aligned}
C= & \sum_{n=1}^{N}\left\{P_{n, 0} \int_{0}^{\eta \bar{\gamma}_{n}} \log _{2}(1+x) \frac{f_{\gamma_{n}}(x)}{F_{\gamma_{n}}\left(\eta \bar{\gamma}_{n}\right)} d x\right. \\
& \left.+P_{n, 1} \int_{\eta \bar{\gamma}_{n}}^{+\infty} \log _{2}(1+x) \frac{f_{\gamma_{n}}(x)}{1-F_{\gamma_{n}}\left(\eta \bar{\gamma}_{n}\right)} d x\right\}
\end{aligned}
$$


where $f_{\gamma_{n}}(x) / F_{\gamma_{n}}\left(\eta \bar{\gamma}_{n}\right)$ and $f_{\gamma_{n}}(x) /\left(1-F_{\gamma_{n}}\left(\eta \bar{\gamma}_{n}\right)\right)$ are the conditional pdfs of $\gamma_{n}$ given $0 \leq \gamma_{n}<\eta \bar{\gamma}_{n}$ and $\gamma_{n} \geq \eta \bar{\gamma}_{n}$, respectively.

In the following, we calculate $P_{n, 0}$ and $P_{n, 1}$. Let $\alpha_{i}(\eta)$ be the probability that $\Gamma_{i} \geq \eta$, which is given by

$$
\alpha_{i}(\eta)=\operatorname{Pr}\left\{\Gamma_{i}=\frac{\gamma_{i}}{\bar{\gamma}_{i}} \geq \eta\right\}=1-F_{\gamma_{i}}\left(\eta \bar{\gamma}_{i}\right) .
$$

Let $\omega_{i}$ denote the channel state of user $i$ as follows:

$$
\omega_{i}= \begin{cases}1, & \Gamma_{i} \geq \eta \\ 0, & \text { otherwise }\end{cases}
$$

Let $\phi=\left(\omega_{1}, \omega_{2}, \ldots, \omega_{N}\right)$, then the probability mass distribution of $\phi$ (i.e., the channel states of $N$ users) is given by

$$
\operatorname{Pr}\{\phi\}=\prod_{i=1}^{N}\left(\alpha_{i}(\eta)\right)^{\omega_{i}}\left(1-\alpha_{i}(\eta)\right)^{1-\omega_{i}} .
$$

Assuming that there are $m$ (excluding user $n$ ) out of $N$ users whose scheduling metrics are above $\eta$, we define the distribution sets of the channel states of $N$ users as follows:

$$
\Omega_{l}(m)=\left\{\phi \mid \omega_{n}=l, \sum_{i=1, i \neq n}^{N} \omega_{i}=m\right\}, l \in\{0,1\}
$$

$P_{n, 0}$ and $P_{n, 1}$ are then given by

$$
P_{n, 0}=\sum_{m=0}^{N-1} \sum_{\phi \in \Omega_{0}(m)}\left[\operatorname{Pr}\{\phi\} \frac{1}{N}\left(1-P_{S}\{\phi\}\right)^{K}\right]
$$

and

$$
\begin{aligned}
P_{n, 1}= & \sum_{m=0}^{N-1} \sum_{\phi \in \Omega_{1}(m)}\left[\operatorname{Pr}\{\phi\}\{\underbrace{\frac{1}{m+1}\left[1-\left(1-P_{S}\{\phi\}\right)^{K}\right]}_{\text {first term }}\right. \\
& +\underbrace{\frac{1}{N}\left(1-P_{S}\{\phi\}\right)^{K}}_{\text {second term }}\}]
\end{aligned}
$$

where $P_{S}\{\phi\}$ denotes the probability that only one user feeds back in one of the $K$ minislots based on a given $\phi \in \Omega_{l}(m)$, and it is given by

$$
P_{S}\{\phi\}=(m+l) p(1-p)^{m+l-1} .
$$

In (8), the first term in the summation denotes the probability that $\Gamma_{n} \geq \eta$ and user $n$ is randomly selected among the successful feedback users, and the second term in the summation denotes the probability that $\Gamma_{n} \geq \eta$ and user $n$ is randomly selected among all users due to no successful feedback in any minislot.

Following the above derivation procedure, we can also obtain the average system fairness and system capacity for the MT scheduling. The only difference is that in (6), $\alpha_{i}(\eta)$ is replaced with $\operatorname{Pr}\left\{\Gamma_{i}=\gamma_{i} \geq \eta\right\}=1-F_{\gamma_{i}}(\eta)$ and, in (5), all $\eta \bar{\gamma}_{n}$ is replaced with $\eta$.

\section{B. Rayleigh Fading Channels}

For Rayleigh fading channels, $\left\{\gamma_{i}\right\}$ are exponentially distributed with the pdf given by

$$
f_{\gamma_{i}}(x)=\frac{1}{\bar{\gamma}_{i}} \exp \left(-\frac{x}{\bar{\gamma}_{i}}\right), \quad x \geq 0
$$

For the PF scheduling, it can be seen from (6) that

$$
\alpha_{1}(\eta)=\alpha_{2}(\eta)=\cdots=\alpha_{N}(\eta) .
$$

As a result, the probability of any user being selected is $1 / N$ regardless of its average SNR. Therefore, strict fairness (i.e., $\zeta=1)$ is achieved by PF scheduling.

The system capacity (5) can be expressed as follows:

$$
\begin{aligned}
C=\frac{1}{N} \sum_{n=1}^{N}\{ & \frac{e^{1 / \bar{\gamma}_{n}}}{\ln 2}\left[e^{\eta} \operatorname{Ei}\left(-\eta-\bar{\gamma}_{n}^{-1}\right)-\operatorname{Ei}\left(-\bar{\gamma}_{n}^{-1}\right)\right] \\
& \left.-\log _{2}\left(1+\bar{\gamma}_{n} \eta\right)\right\} \\
& \cdot \sum_{m=0}^{N-1}\left\{\left(\begin{array}{c}
N-1 \\
m
\end{array}\right) e^{-m \eta}\left(1-e^{-\eta}\right)^{N-m-1}\right. \\
& \left.\cdot\left[1-m p(1-p)^{m-1}\right]^{K}\right\} \\
+ & \frac{1}{N} \sum_{n=1}^{N}\left[\log _{2}\left(1+\bar{\gamma}_{n} \eta\right)-\frac{e^{1 / \bar{\gamma}_{n}+\eta}}{\ln 2} \operatorname{Ei}\left(-\eta-\bar{\gamma}_{n}^{-1}\right)\right]
\end{aligned}
$$

where $\operatorname{Ei}(\cdot)$ is the exponential integral function defined by $\operatorname{Ei}(x)=\int_{-\infty}^{x} e^{t} / t d t$.

\section{The Optimization of $p$ and $\eta$}

The system capacity $C(p, \eta)$ can be maximized by optimizing the parameters of $p$ and $\eta$. If the number of active users $N$ and the number of minislots $K$ are known by the BS, the optimization problem can be expressed as follows:

$$
\begin{array}{ll} 
& \max _{p, \eta} C(p, \eta) \\
\text { s.t. } & 0 \leq p \leq 1, \quad \eta \geq 0 .
\end{array}
$$

The optimization problem may not be convex. The constrained gradient descent method [11] can be used to find a locally optimum solution to the problem (12) given that the distributions of $\left\{\gamma_{i}\right\}$, i.e., $f_{\gamma_{i}}(x)$, are available at the BS. $f_{\gamma_{i}}(x)$ can be estimated at the BS dynamically by collecting the uplink feedback information. It can be seen from (5), (7) and (8) that given $f_{\gamma_{i}}(x)$, the partial derivatives of system capacity (5) with respect to $p$ and $\eta$ (i.e., $\partial C / \partial p$ and $\partial C / \partial \eta$ ) can be evaluated in closed-form. As a result, the implementation of the constrained gradient descent method is straightforward.

To use the method, it is important to select proper initial values. For Rayleigh fading channels, we select the initial values as follows. Instead of searching $\eta$, we search $q=e^{-\eta}$ (thus $0 \leq q \leq 1$ and $\eta=-\ln q$ ). For the system configuration described in Section IV, we initialize the $(p, q)$ at $\left(0.5, e^{-10^{0.3}}\right)$ and $\left(0.5, e^{-10^{0.9}}\right)$ for PF and MT schedulings, respectively. Table I gives the optimal $p$ and $\eta$ maximizing system capacity by using the constrained gradient descent method [11], whose optimality is verified through exhaustive search. 
TABLE I

OPTIMAL VALUES OF $p$ AND $\eta$

\begin{tabular}{|c|c|c|c|c|}
\hline & \multicolumn{2}{|c|}{ PF scheduling } & \multicolumn{2}{c|}{ MT scheduling } \\
\hline$N$ & $p$ & $\eta(\mathrm{dB})$ & $p$ & $\eta(\mathrm{dB})$ \\
\hline 6 & 0.545 & 1.37 & 0.618 & 8.17 \\
\hline 18 & 0.436 & 3.41 & 0.456 & 10.44 \\
\hline 30 & 0.403 & 4.16 & 0.425 & 11.53 \\
\hline 42 & 0.385 & 4.60 & 0.417 & 12.08 \\
\hline 54 & 0.372 & 4.91 & 0.388 & 12.32 \\
\hline 66 & 0.363 & 5.14 & 0.345 & 12.53 \\
\hline
\end{tabular}

\section{NUMERICAL RESULTS}

Fairness and capacity performances are evaluated for the opportunistic feedback protocol with PF or MT scheduling under a Rayleigh fading channel environment. We divide all users (the total number of the users is a multiple of three) into three groups. Each group has the same number of users, and the users within the same group have the same average SNR. The average SNR values for the three groups are $3 \mathrm{~dB}, 6 \mathrm{~dB}$ and $9 \mathrm{~dB}$, respectively. The number of minislots, $K$, is fixed to be 4 . The numerical results of the full feedback protocol in [3] are presented as performance benchmarks, and the fixedslot polling (i.e., the BS randomly polls a small subset of users, then selects the user with the best channel from these users) is also considered for performance comparison. For the simulations of the fixed-slot polling, the subset size is set to be $K$.

Fig. 2 shows the average system fairness versus the number of users. The average system fairness curves of PF scheduling for both the full feedback protocol and opportunistic feedback protocol overlap. As expected, the PF scheduling achieves strict fairness, whereas the MT scheduling demonstrates low fairness since the users with higher average SNRs have greater opportunities of competing for the feedback minislots. As also shown in Fig. 2, the opportunistic feedback protocol offers better average system fairness than that of the full feedback protocol in case of the MT scheduling because the former allows the users with scheduling metrics above the threshold to be selected with equal probability, while the latter only selects the user with the highest SNR.

Fig. 3 shows the system capacity versus the number of users. The system capacity of PF scheduling is lower than that of MT scheduling. For example, there is a performance loss of about $10 \%$ for opportunistic feedback protocol. Although the opportunistic feedback protocol suffers from a performance loss compared with full feedback protocol, it only uses a fixed number of feedback minislots regardless of the number of users. In contrast, the full feedback protocol requires feedback overhead (including not only all UID information, but also all users' CQI) that increases linearly with the number of users. From Figs. 2 and 3, it can be seen that the opportunistic feedback protocol with PF scheduling outperforms the fixed-slot polling significantly in both fairness and capacity performances when the number of active users is reasonably large.

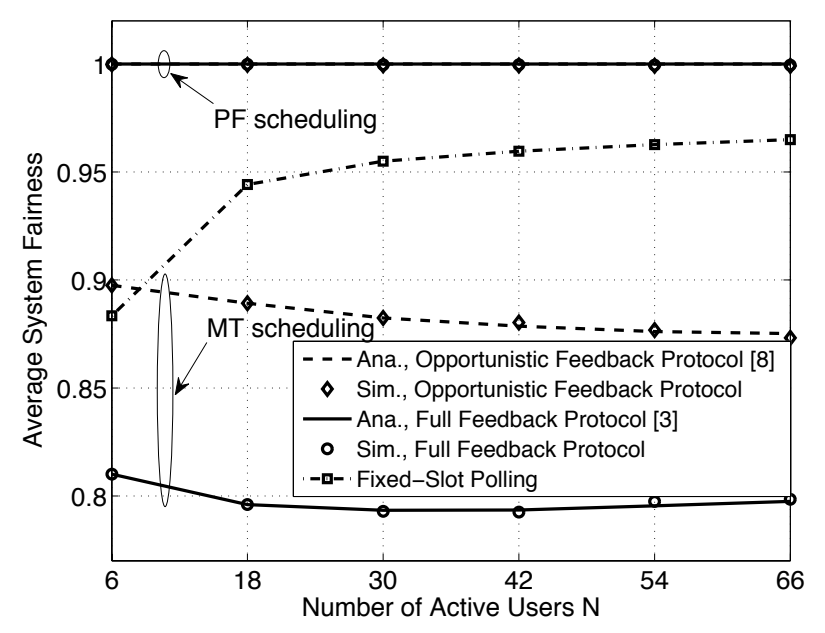

Fig. 2. Average system fairness versus the number of users.

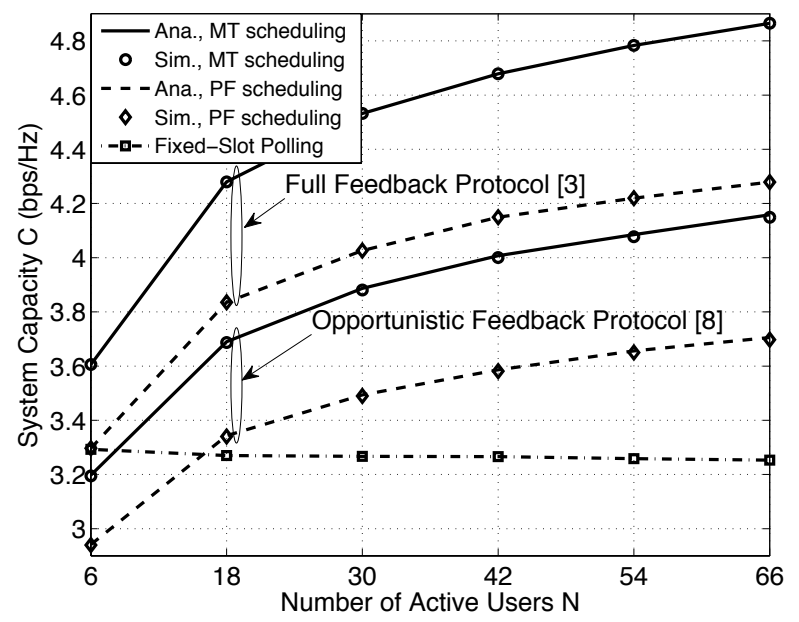

Fig. 3. System capacity versus the number of users.

\section{CONCLUSION}

The performances of both average system fairness and system capacity are analyzed and compared for the opportunistic feedback protocol with PF or MT scheduling. It has been shown that the PF opportunistic feedback protocol achieves strict fairness at the cost of a small loss of system capacity over Rayleigh fading channels, compared with MT scheduling. In addition, the constrained gradient descent method is used to search the feedback threshold and random access probability of the opportunistic feedback protocol.

\section{REFERENCES}

[1] R. Knopp and P. A. Humblet, "Information capacity and power control in single-cell multiuser communications," in Proc. IEEE ICC'95, vol. 1, Seattle, Jun. 1995, pp. 331-335.

[2] D. N. C. Tse, "Optimal power allocation over parallel gaussian channels," in Proc. ISIT, Ulm, Germany, Jun. 1997, p. 27.

[3] L. Yang, M. Kang, and M.-S. Alouini, "On the capacity-fairness tradeoff in multiuser diversity systems," IEEE Trans. Veh. Technol., vol. 56, no. 4, pp. 1901-1907, Jul. 2007. 
[4] P. Viswanath, D. N. C. Tse, and R. Laroia, "Opportunistic beamforming using dumb antennas," IEEE Trans. Inf. Theory, vol. 48, no. 6, pp. 1277 1294, Jun. 2002.

[5] D. Gesbert and M. Slim-Alouini, "How much feedback is multi-user diversity really worth?" in Proc. Int. Conf. on Commun., Jun. 2004, pp. 234-238

[6] O. Somekh, A. Haimovich, and Y. Bar-Ness, "Sum-rate analysis of downlink channels with 1-bit feedback," IEEE Commun. Lett., vol. 11, no. 2, pp. 137-139, Feb. 2007.

[7] N. Jindal, "MIMO broadcast channels with finite-rate feedback," IEEE Trans. Inf. Theory, vol. 52, no. 11, pp. 5045-5060, Nov. 2006.

[8] T. Tang and R. W. Heath, "Opportunistic feedback for downlink multiuser diversity," IEEE Commun. Lett., vol. 9, no. 10, pp. 948-950, Oct. 2005.

[9] H. Li, Q. Guo, and D. Huang, "Throughput analysis of opportunistic feedback for downlink multiuser diversity with capture effect," IEEE Commun. Lett., vol. 16, no. 1, pp. 44-46, Jan. 2012.

[10] J. So and J. M. Cioffi, "Capacity and fairness in multiuser diversity systems with opportunistic feedback," IEEE Commun. Lett., vol. 12, no. 9, pp. 648-650, Sep. 2008.

[11] S. Boyd and L. Vandenberghe, Convex Optimization. Cambridge, U.K.: Cambridge Univ. Press, 2004.

[12] J.-G. Choi and S. Bahk, "Cell-throughput analysis of the proportional fair scheduler in the single-cell environment," IEEE Trans. Veh. Technol., vol. 56, no. 2, pp. 766-778, Mar. 2007.

[13] R. Elliott, "A measure of fairness of service for scheduling algorithms in multiuser systems," in Proc. IEEE CCECE, Winnipeg, MB, Canada, May 2002, pp. 1583-1588.

[14] T. Kim and J.-T. Lim, "Capacity and fairness tradeoff in multiuser scheduling system with reduced feedback," IEEE Commun. Lett., vol. 13, no. 11 , pp. 841-843, Nov. 2009. 